\title{
Algorithm for fringe pattern normalization
}

\author{
Juan Antonio Quiroga *, José Antonio Gómez-Pedrero, Ángel García-Botella \\ Departamento de Optica de la Universidad Complutense, Facultad de CC. Físicas. Ciudad Universitaria S/N, Madrid 28040, Spain \\ Received 18 May 2001; received in revised form 4 July 2001; accepted 16 July 2001
}

\begin{abstract}
In this work we present a new algorithm for fringe pattern normalization, that is, background suppression and modulation normalization. Normalization is necessary for several fringe pattern processing techniques. For example, this is the case of the regularization and phase sampling methods. In general, background suppression can be accomplished by high-pass filtering, however if modulation is not constant or almost constant over the field of view, normalization is a difficult task. The solution proposed is based in the use of two orthogonal bandpass filters, from which a normalized irradiance distribution is obtained. We have applied the method to simulated as well as experimental data with good results. (c) 2001 Published by Elsevier Science B.V.
\end{abstract}

Keywords: Fringe pattern processing; Optical metrology; Fourier transform; Background suppression; Normalization

\section{Introduction}

During the last years many methods for fringe pattern analysis have been developed. The main objective of these methods is the extraction of the information contained in a single or various fringe patterns. Normally this information is contained in the modulating phase, that depending on the particular set-up, can be associated to a given physical magnitude as deformation, shape, temperature, index gradients, etc.

Most of the fringe pattern analysis methods assume a sinusoidal model for the irradiance of the fringe pattern, that is,

$i(\mathbf{r})=b(\mathbf{r})+m(\mathbf{r}) \cos (\phi(\mathbf{r}))$,

\footnotetext{
${ }^{*}$ Corresponding author. Tel.: +34-91-394-45-55; fax: +34-91394-46-78.

E-mail address: aq@fis.ucm.es (J.A. Quiroga).
}

where $i$ is the irradiance, $b$ the background, $m$ the modulation, $\phi$ the modulating phase and $\mathbf{r}$ denotes the spatial dependence of these quantities.

In the basic version of the phase sampling techniques [1], a set of known phase steps $\alpha_{n}$ are introduced in the modulating phase and a set of irradiances $i_{n}(\mathbf{r})=b(\mathbf{r})+m(\mathbf{r}) \cos \left(\phi(\mathbf{r})+\alpha_{n}\right)$ are acquired. Finally the tangent of the phase is computed by

$\tan \phi=\frac{i_{\mathrm{S}}}{i_{\mathrm{C}}}$,

where $i_{\mathrm{S}}$ and $i_{\mathrm{C}}$ denote the irradiances associated to the sine and cosine of the modulating phase multiplied by the modulation, that is

$$
\begin{aligned}
& i_{\mathrm{S}} \approx m \sin \phi, \\
& i_{\mathrm{C}} \approx m \cos \phi,
\end{aligned}
$$

and they are always computed as a suitable combination of the phase shifted images $i_{n}$. 
The quality of the approximation represented by Eq. (3) determines the error of the phase obtained by Eq. (2). In this class of algorithms there is an important assumption: the background and the modulation do not change with the phase step. Normally this is fulfilled, however when problems as a lack of illumination control appear, using directly Eq. (2) generates big errors in the demodulated phase.

Another possibility for phase demodulation is the regularized phase tracking method (RPT) [2]. The basic idea of this technique is that locally the intensity of the fringe pattern may be considered as spatially monochromatic, so its irradiance may be modelled as a sinusoidal function phase modulated by a plane $p(\cdot)$. Thus by minimizing an appropriate cost function that takes into account the differences between the measured irradiance and the proposed model it will be possible to demodulate the phase $\phi(\mathbf{r})$ from the irradiance $i(\mathbf{r})$. In the standard RPT the cost function to be minimized at each site $\mathbf{r}=(x, y)$ is:

$$
\begin{aligned}
U(\mathbf{r})= & \sum_{(\xi, \eta) \in\left(N_{\mathbf{r}} \cap S\right)}\left\{\left[i^{\prime}(\mathbf{r})-\cos p(x, y, \xi, \eta)\right]^{2}\right. \\
& \left.+\beta\left[\phi_{0}(\xi, \eta)-p(x, y, \xi, \eta)\right]^{2} m(\xi, \eta)\right\},
\end{aligned}
$$

where

$$
\begin{gathered}
p(x, y, \xi, \eta)=\phi_{0}(x, y)+\omega_{x}(x, y)(x-\xi) \\
+\omega_{y}(x, y)(y-\eta)
\end{gathered}
$$

is the predictor plane, $S$ denotes the region where valid data is present; $N_{\mathbf{r}}$ is the neighbourhood region around the site $\mathbf{r}=(x, y)$ where the phase is being estimated; $m(\mathbf{r})$ is an indicator field which equals one if the phase at site $\mathbf{r}$ has already been estimated, and zero otherwise; the fringe pattern $i^{\prime}(\mathbf{r})$ is the normalized version of $i(\mathbf{r})$. The fields $\omega_{x}(\mathbf{r})$ and $\omega_{y}(\mathbf{r})$ are the estimated local frequencies along the $x$ and $y$ directions respectively. Finally the parameter $\beta$ is a constant denominated regularization parameter that controls (along with the size of $\left.N_{\mathbf{r}}\right)$ the smoothness of the detected phase $\phi_{0}(\mathbf{r})$.

To demodulate the phase from a given fringe pattern with the RPT method we need to minimize the cost function $U(\mathbf{r})$ at each point with respect to the variables $\phi_{0}(\mathbf{r}), \omega_{x}(\mathbf{r})$ and $\omega_{y}(\mathbf{r})$. In this case, (a)

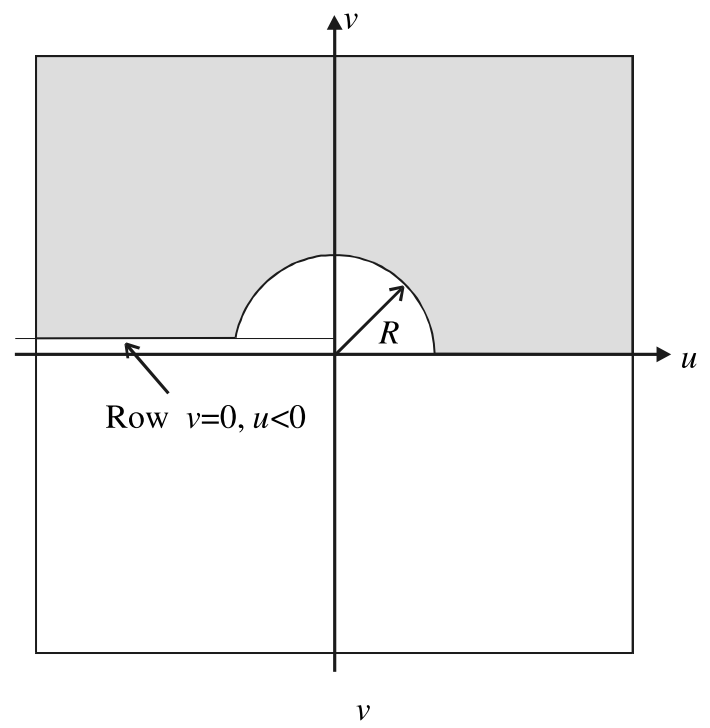

(b)

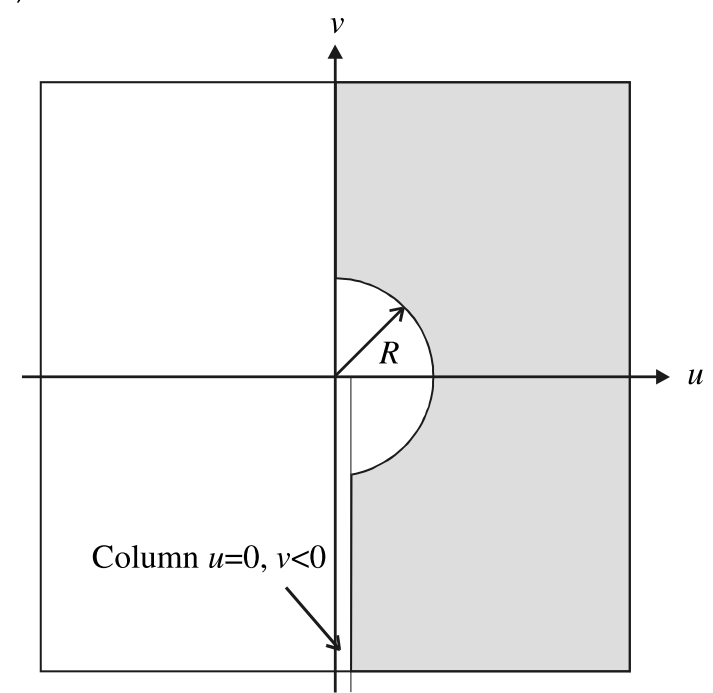

Fig. 1. (a) Scheme of the filter $h_{1}(\mathbf{r})$ and (b) corresponding scheme for $h_{2}(\mathbf{r})$. In these pictures, dark zones corresponds with the filtered regions of the Fourier spectrum. As it is shown in these diagrams, both filters are the combination of a bidimensional bandpass filter for the $+v$ plane, in the case of $h_{1}(+u$ plane in the case if $h_{2}$ ), and a circular high-pass filter of radius $R$ (see text for details).

any deviation of $i^{\prime}(\mathbf{r})$ from a normalized irradiance will made this minimization process tend to fail.

From the above mentioned reasons it is clear that a fast normalization algorithm could be of 
great utility as a pre-processing tool to be employed by several fringe processing techniques. Therefore, we propose in this work a direct method that can be briefly described as follows: given the irradiance signal, we perform first a filter operation using two orthogonal bandpass filters. In this way, we obtain two analytical signals whose respective phases can be combined in order to get an estimation of $i^{\prime}(\mathbf{r})$. The filtering process can be implemented as a Fourier transform operation, so fast transforms are applicable to this problem.

This work is organized as follows: in Section 2 we present the theoretical foundations of the method. In Section 3 we apply the normalization algorithm to a real fringe pattern and finally conclusions are given in Section 4.

\section{Theoretical foundations}

The algorithm designed for irradiance normalization is based in the use of two orthogonal bandpass filters. Following the original work of Kreis et al. [3], the first bandpass filter, $h_{1}(\mathbf{r})$, is designed to filter out all the negative horizontal spatial frequencies eliminating at the same time the low frequencies in order to remove the background. In consequence, its frequency response is given by $H_{1}(u, v)=H(v) \operatorname{CHP}(R)$, where $H(v)$ is a bidimensional bandpass filter for the $+v$ half plane

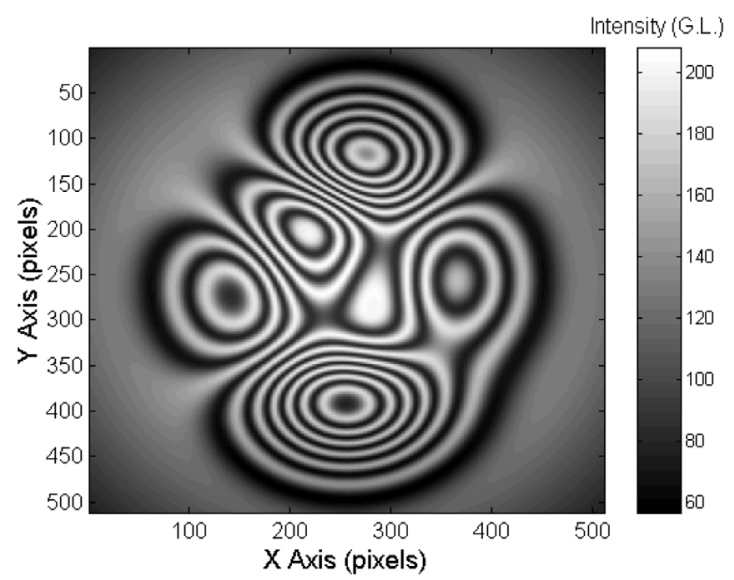

Fig. 2. Simulated fringe pattern image used to test numerically the normalization algorithm. Image size is $512 \times 512$ pixels and the intensity is coded between 60 and 210 grey levels (GL). (a)

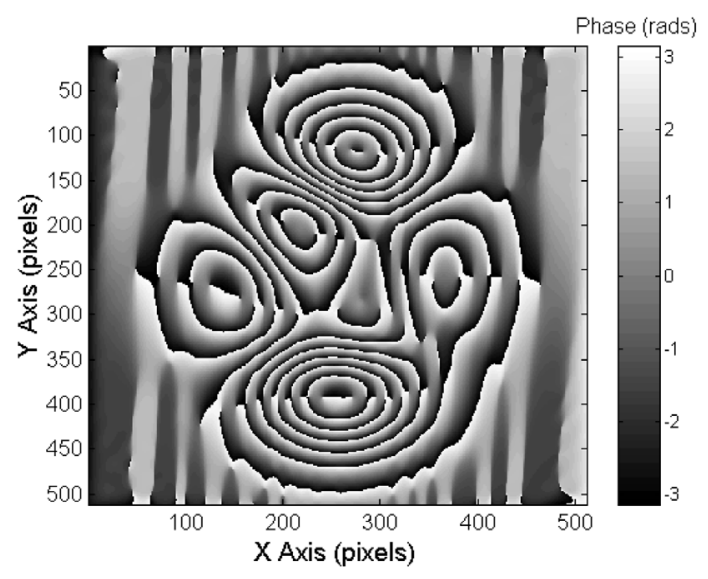

(b)

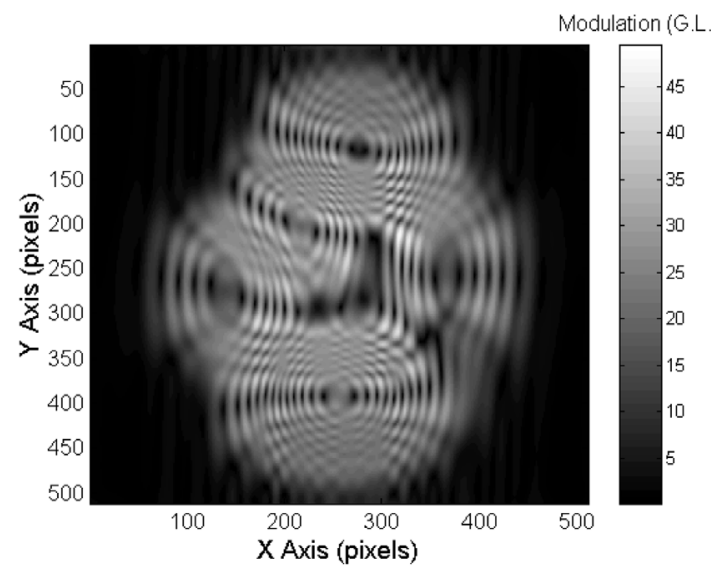

Fig. 3. (a) Phase of the analytical signal obtained when filter $h_{1}(\mathbf{r})$ is applied to the fringe pattern distribution of Fig. 2, (b) modulation corresponding to this analytic signal.

(the bidimensional equivalent of the Heaviside unit step function [4]) and $\operatorname{CHP}(R)$ is a circular highpass filter of radius $R$ (see Fig. 1a). Thus the application of this filter to the irradiance singal (1) gives a complex signal like

$i_{1}(\mathbf{r})=i(\mathbf{r}) \otimes h_{1}(\mathbf{r})=m_{1}(\mathbf{r}) \exp \left(\mathrm{i} \phi_{1}\right)$,

where $\otimes$ denotes a convolution product. The phase of this analytic signal, $\phi_{1}$, will present sign discontinuities (equivalent to a $\pi$ phase jump) every time the local tangent of the fringes becomes horizontal. Thus the corresponding modulation, 
(a)

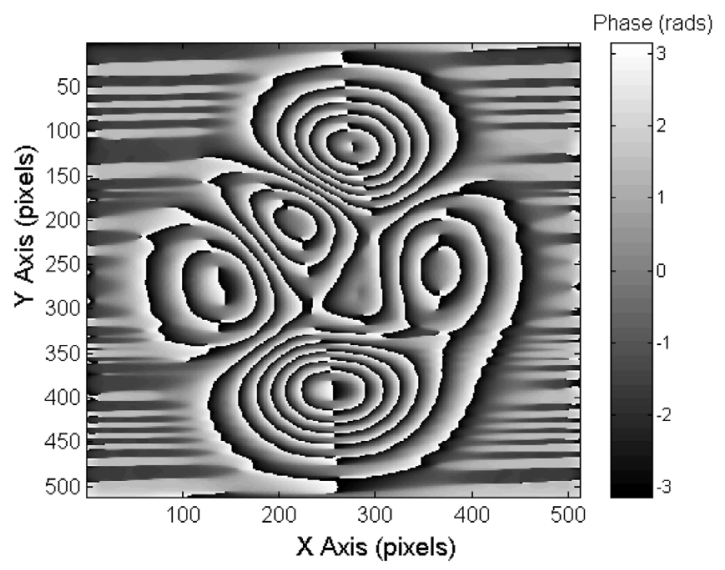

(b)

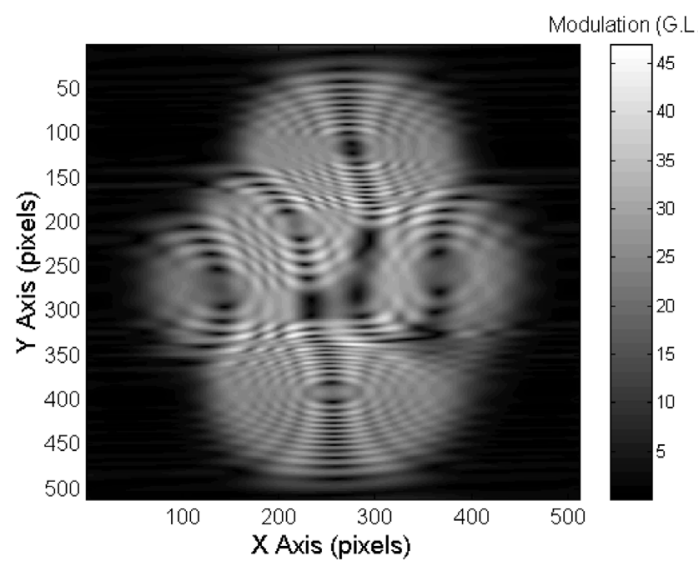

Fig. 4. In this figure, we represent the phase (a) and modulation (b) of the analytical signal obtained after application of filter $h_{2}(\mathbf{r})$ over the fringe pattern image represented in Fig. 2.

$m_{1}$, will be very low at those points were the isophasic lines are horizontal. As an example Fig. 2 shows a simulated fringe pattern and Fig. 3 shows the modulation and the phase of the $i_{1}$ signal corresponding to the fringe pattern of Fig. 2.

The second bandpass filter, $h_{2}(\mathbf{r})$, is designed to filter out all the negative vertical spatial frequencies eliminating at the same time the low frequencies in order to remove the background, in consequence its frequency response is $H_{2}(u, v)=$ $H(u) \mathrm{CHP}(R)$ (see Fig. 1b), where $H(u)$ is a bidimensional bandpass filter for the $+u$ half plane. The result of apply this filter to the irradiance signal (1) is a complex signal, given by

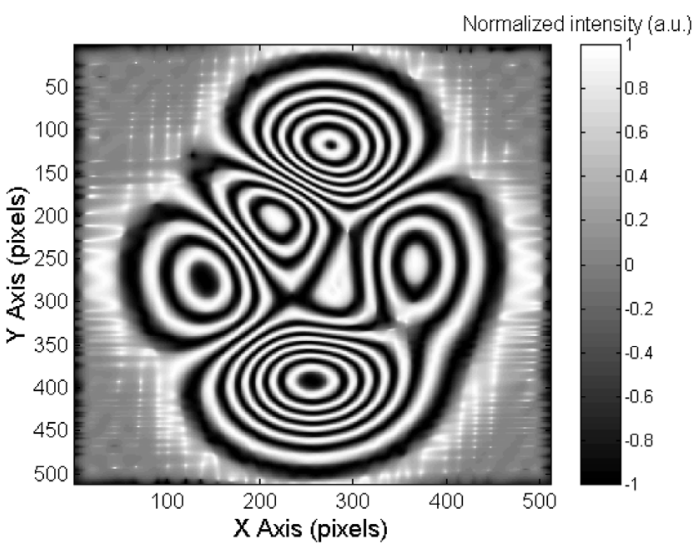

Fig. 5. Normalized fringe pattern corresponding to Fig. 2 obtained with the proposed method with $R=17$ fringes/field.

(a)

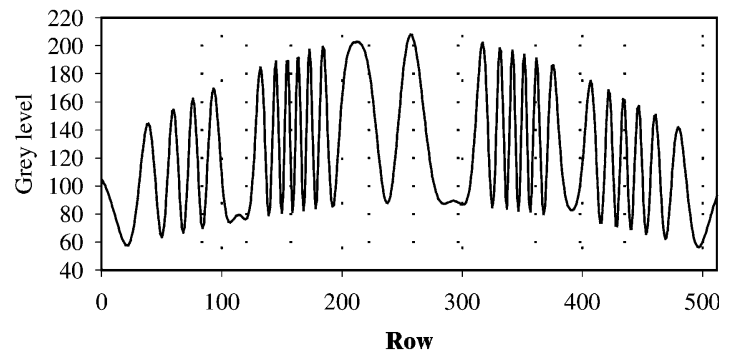

(b)

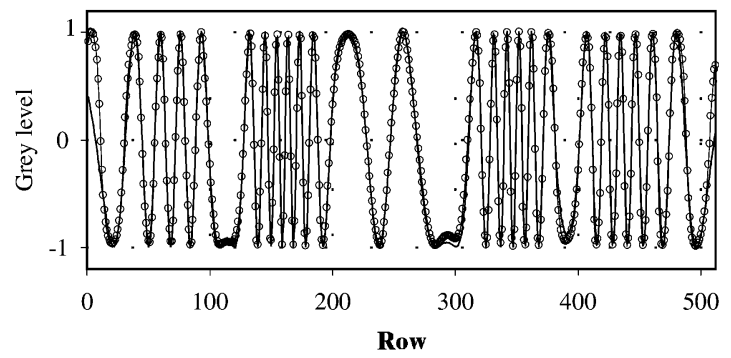

Fig. 6. (a) Intensity profile along the column 250 of the simulated irradiance distribution, (b) comparison between the normalized intensity profiles corresponding to column 250 of the simulated irradiance distribution with the theoretical result (cosine of the modulating phase of the irradiance pattern depicted in Fig. 2).

$i_{2}(\mathbf{r})=i(\mathbf{r}) \otimes h_{2}(\mathbf{r})=m_{2}(\mathbf{r}) \exp \left(\mathrm{i} \phi_{2}\right)$.

The phase of this analytic signal, $\phi_{2}$, will present sign discontinuities at those points were the 
isophasic lines have vertical tangent. Thus the modulation (in this case $m_{2}$ ) will be very low at the points in which the isophasic lines are vertical. Fig. 4 shows the modulation and the phase of the $i_{2}$ signal corresponding to the fringe pattern of Fig. 2. For Figs. 3 and 4 the size of the image was $512 \times 512$ pixels and the radius $R$ was 17 fringes/ field.

In principle, either $\phi_{1}$ or $\phi_{2}$, can be used to obtain a normalized version of the irradiance pattern (1) just by computing their cosine (the use of the sine is precluded because of its odd character). However these cosine images will have areas where severe distortions will be present due to the low-modulation areas existent in $i_{1}$ or $i_{2}$.

The solution adopted uses the fact that the lowmodulation areas of $i_{1}$ or $i_{2}$ are in quadrature, that is, they never superpose, as can be observed in Figs. 3 and 4 (there are no places with vertical and horizontal fringes at the same time). Using this

(a)

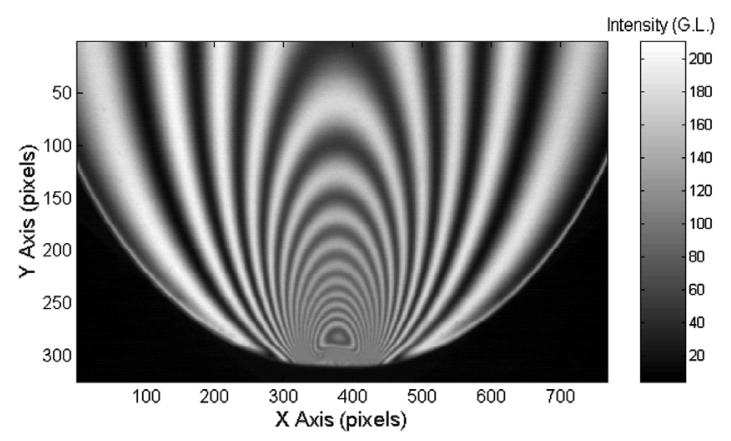

property, the solution that we have finally adopted is a weighted average of the cosine of $\phi_{1}$ or $\phi_{2}$ being the weights the modulations $m_{1}$ and $m_{2}$ that is

$$
\begin{aligned}
i^{\prime}(\mathbf{r}) & =\cos (\phi(\mathbf{r})) \\
& \approx \frac{m_{1}(\mathbf{r}) \cos \left(\phi_{1}(\mathbf{r})\right)+m_{2}(\mathbf{r}) \cos \left(\phi_{2}(\mathbf{r})\right)}{m_{1}(\mathbf{r})+m_{2}(\mathbf{r})} .
\end{aligned}
$$

The result of applying Eq. (7) to the irradiance distribution shown in Fig. 2 is shown in Fig. 5. Fig. 6a shows a profile along column 250 of the fringe pattern shown in Fig. 2. A comparison for the same profile between the normalized irradiance of Fig. 5 and the theoretical result is shown in Fig. 6b (the theoretical result is obtained just by calculating the cosine of the modulating phase of the fringe pattern depicted in Fig. 2).

There are two practical aspects that should be taken into account in order to apply properly Eq. (7). The first one deals with the structure of $H_{1}$ and

(b)

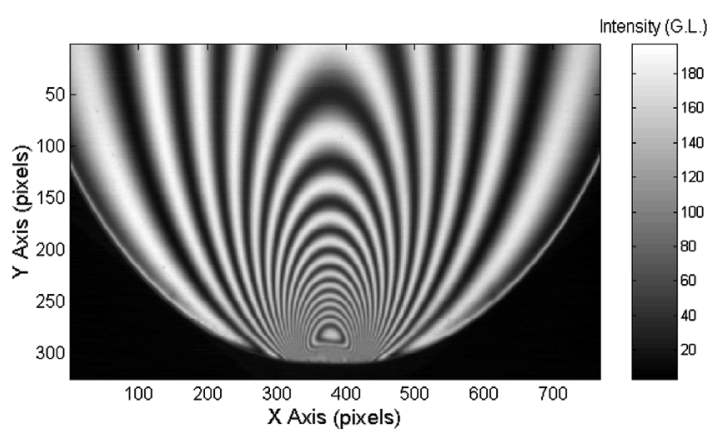

(c)

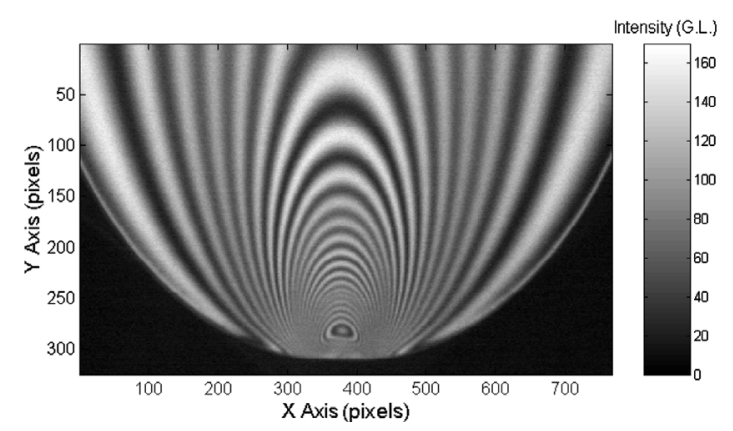

Fig. 7. (a) Intensity distribution associated with the $\mathrm{R}$ band of a isochromatic fringe pattern corresponding to a diametrically loaded disk, (b) and (c) are the fringe patterns that correspond to the $\mathrm{G}$ and $\mathrm{B}$ channels, respectively. 
$\mathrm{H}_{2}$ for the frequencies $v=0$ and $u=0$, respectively. As the irradiance signal is real, its spectrum will be hermitian, that is, if $I(u, v)$ is the Fourier transform of $i(\mathbf{r})$ then $I(u, v)=\tilde{I}(-u,-v)$, were $\sim$ denotes the complex conjugate operation. Following Kreis et al. [3] the final objective of the $H_{1}$ and $\mathrm{H}_{2}$ filters is to break the hermitian character of the fringe pattern spectrum by setting to zero either $I(u, v)$ or $I(-u,-v)$ for each spatial frequency. This makes the design of the $H_{1}$ and $H_{2}$ filters asymmetrical with respect the origin, as shown in Fig. 1.

The second aspect relates with the main assumption of Eq. (7): the low-modulation areas of $I_{1}$ and $I_{2}$ are in quadrature. This is true whenever fringes are present, or that is the same, there is

(a)

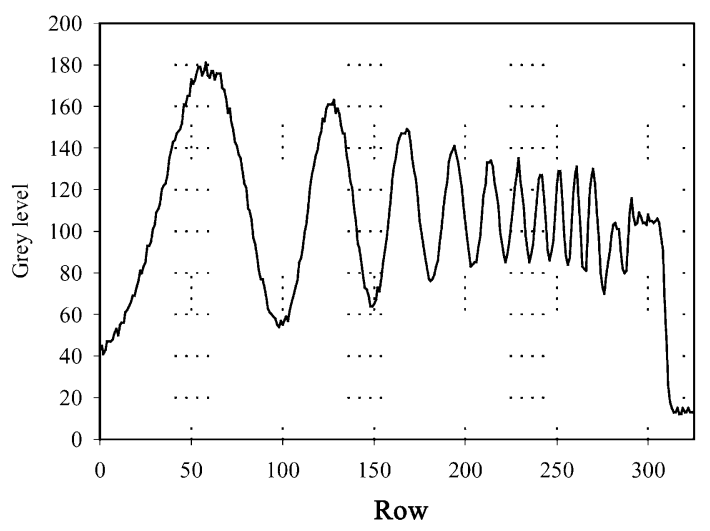

spatial frequency content. The areas with no fringes (extreme of the phase or constant phase zones) act as a distortion sources in Eq. (7). So, in general the higher the spectral content of a given fringe pattern the better is the normalized approximation (7). Anyway, we must state that it is possible to detect the low-modulation areas by thresholding the total modulation of the normalized fringe pattern computed as the sum of $m_{1}$ and $m_{2}$.

\section{Experimental results}

To show the performance of the algorithm we have chosen a photoelastic experiment. When a transparent stressed sample is placed inside a

(b)

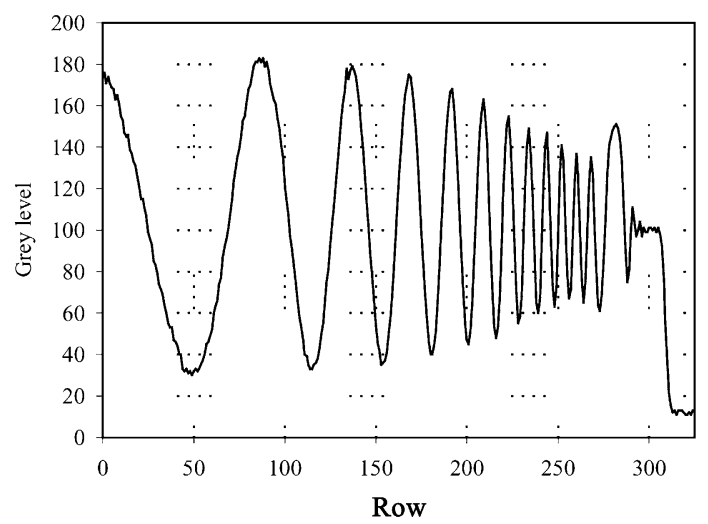

(c)

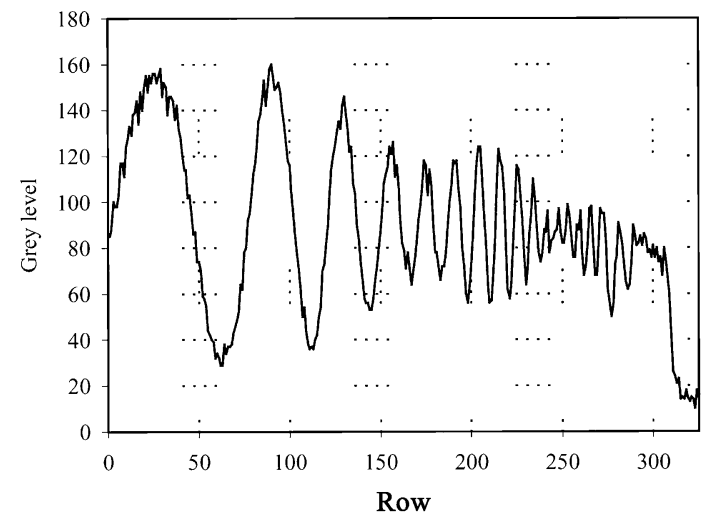

Fig. 8. Intensity profiles along the column 378 of the fringe patterns depicted in Fig. 7. (a) is the intensity profile for the R channel, while the intensity profiles depicted in (b) and (c), correspond to the $\mathrm{G}$ and $\mathrm{B}$ channels, respectively. 
circular polariscope under the circular dark field configuration [5] and the set-up is illuminated with monochromatic illumination the output irradiance can be described by Eq. (5).

In this particular case the fringes are denominated isochromatics and the phase is associated with the difference of principal stresses by

$\phi=\frac{2 \pi}{\lambda} C_{\lambda} d\left(\sigma_{1}-\sigma_{2}\right)$,

where $\lambda$ is the wavelength used, $C_{\lambda}$ is the photoelastic constant for the given wavelength, $d$ is the thickness of the sample and $\sigma_{1}$ and $\sigma_{2}$ are the principal stresses. The variations in the background, $b(\mathbf{r})$, are related to inhomogeneities in the illumination, and the changes in the modulation, $m(\mathbf{r})$, are mainly related with the phase gradients. Due to the finite detector size, if the fringe density increases a low-pass effect appears and this is manifested in form of lack of modulation.

(a)

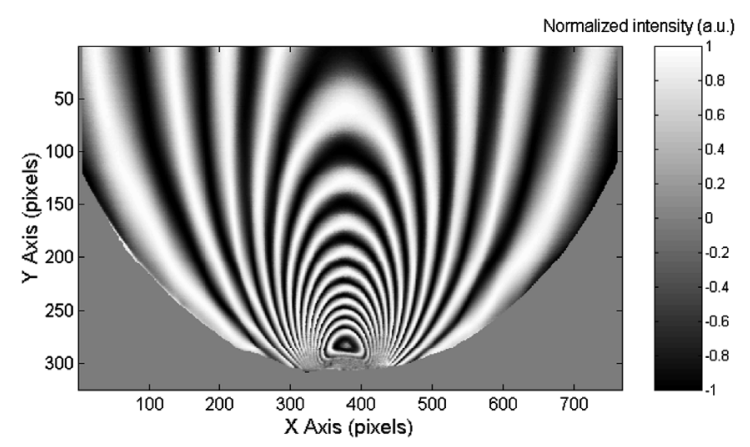

If a discrete spectrum source such a fluorescent lamp is used and the images are captured with a RGB-CCD, due to the spectral response of the RGB filters each of the three bands of the RGB isochromatic fringe pattern obtained can be considered as monochromatic.

Fig. 7 shows the three bands of a RGB isochromatic fringe pattern corresponding to a diametrically loaded disk. In the three cases the lack of modulation is evident near the contact point. In the case of the B channel superposed to this effect it is observable a beat in the modulation of the fringes that indicates a non-monochromatic character. Fig. 8 shows the profiles of the images of Fig. 7 for the central diameter (that corresponds to column 378). This variation in the modulation makes very difficult the demodulation of the images of Fig. 7 by a regularization algorithm.

Fig. 9 shows the normalized irradiances corresponding to Fig. 7, and Fig. 10 shows the same

(b)

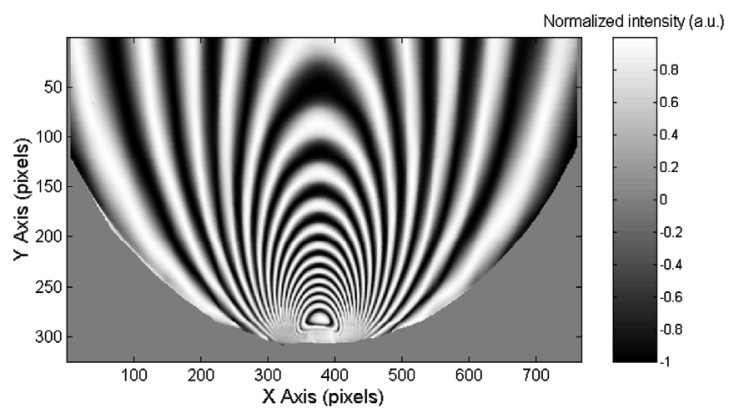

(c)

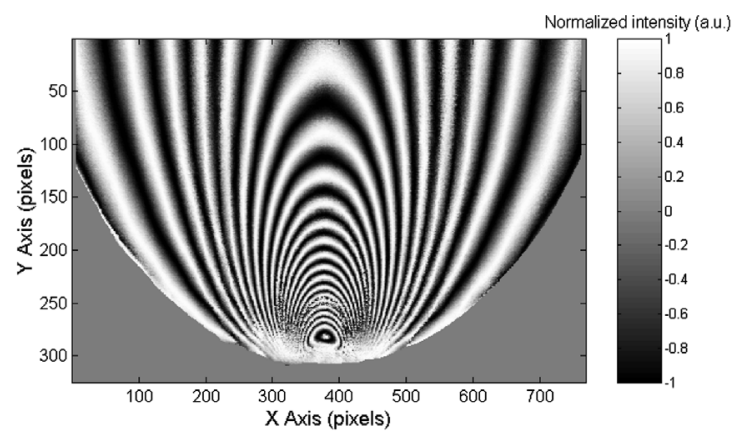

Fig. 9. (a) Normalized intensity distribution associated with the $\mathrm{R}$ band of the isochromatic fringe pattern depicted in Fig. 7. (b) and (c) are the normalized fringe patterns corresponding to the $\mathrm{G}$ and $\mathrm{B}$ channels, respectively. In this case $R=5$ fringes/field. 
(a)

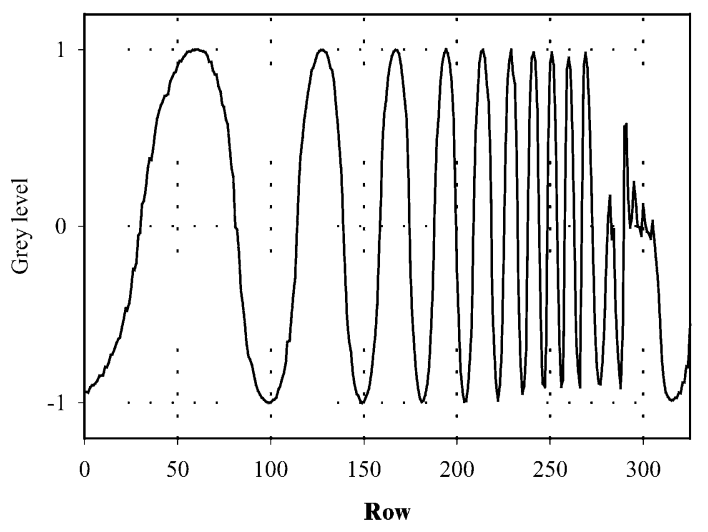

(b)

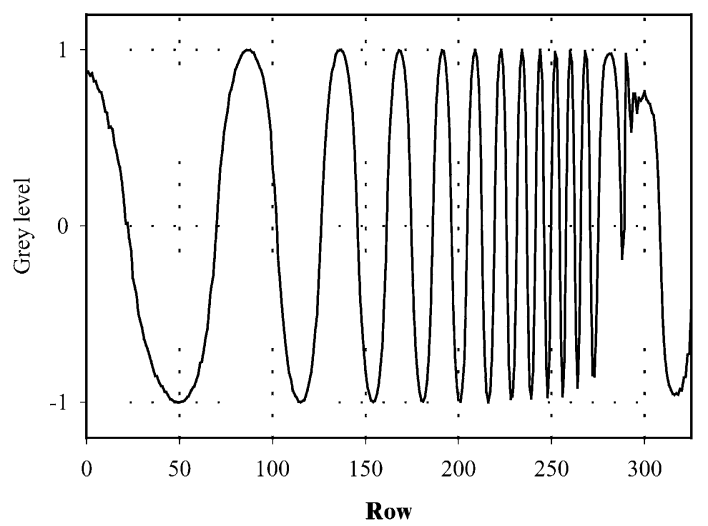

(c)

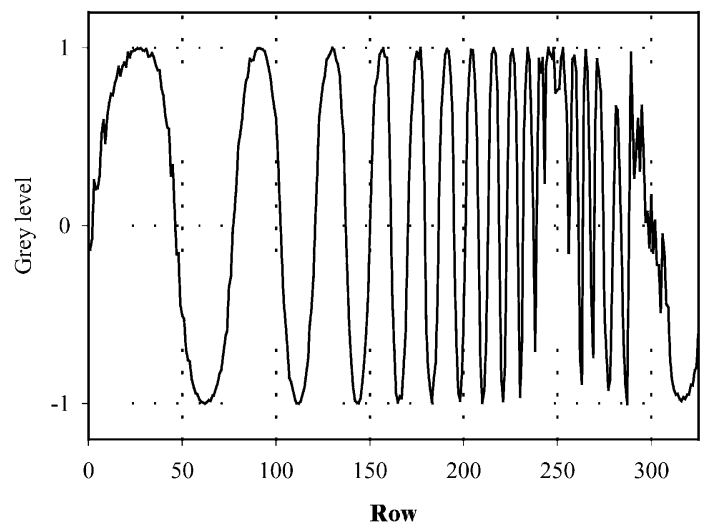

Fig. 10. Normalized intensity profiles along the column 378 of the fringe patterns depicted in Fig. 9. Fig. 9a is the profile for the R channel, while the normalized intensity profiles depicted in Fig. $9 \mathrm{~b}$ and c, correspond to the $\mathrm{G}$ and B channels, respectively.

profiles of Fig. 8, but for the normalized images. The effects of the normalization are evident in these profiles, even for the case of the B channel. In this case the size of the images was $325 \times 768$ and the radius $R$ was 5 fringes/field.

\section{Conclusions}

We have presented a new fringe pattern normalization algorithm useful for techniques as phase sampling and regularization. The algorithm is based in the use of two orthogonal bandpass filters and can be applied a direct and fast method thanks to the possibility of implementing it as a
FFT operation. We have tested the algorithm with real data obtaining good results.

\section{Acknowledgements}

We wish to thank the economical support of this work given by European Union, project INDUCE, BRPR-CT97-0805.

\section{References}

[1] D. Malacara, M. Servín, Z. Malacara, Interferogram Análisis for Optical Testing, Marcel Dekker, New York, 1998. 
[2] M. Servin, J.L. Marroquin, F.J. Cuevas, Demodulation of a single interferogram by use of a two-dimensional regularized phase-tracking technique, Appl. Opt. 36 (1997) 45404548 .

[3] T.M. Kreis, W.P.O. Jüpner, Fourier-transform evaluation of interference patterns: the role of filtering in the spatial frequency domain, Laser Interferometry Quantitative Analysis of Interferograms, Proc. SPIE 1162 (1989) 116-125.

[4] R.N. Bracewell, The Fourier Transform and its Applications, second ed., McGraw-Hill, New York, 1978.

[5] P.S. Teocaris, E.E. Gdoutos, Matrix Theory of Photoelasticity, Springer-Verlag, Berlin, 1979. 